\title{
Development of an Automated Device for the Procedure of Blood Transfusion in Newborns
}

\author{
Patrícia Quariguazy da Frota ${ }^{1}$ Josiane Dantas Viana Barbosa ${ }^{2}$, Paulo Roberto Freitas Neves ${ }^{2}$ \\ ${ }^{1}$ Bahiana School of Medicine and Public Health, ${ }^{2}$ SENAI CIMATEC University Center; Salvador, Bahia, Brazil
}

\begin{abstract}
Hemolytic disease of the newborn, also known as hemolytic disease of the fetus and newborn, HDN, HDFN, is a problem of fetal erythrocyte hemolysis. This may happen due to the sensitization of maternal antibodies through the placental route. It is the pathology most frequently found in neonatal patients. Approximately $98 \%$ of maternal alloimmunization cases by erythrocyte antigens are due to the $\mathrm{RhD}$ factor. Phototherapy is the first choice in the treatment of neonatal jaundice. Blood transfusion is the therapy instituted for the treatment of severe neonatal disease. This study aimed to develop an automated device for performing the blood transfusion procedure. The method was developed in four stages: (1) Literature review about the search for theoretical references based on scientific articles and textbooks on transfusion therapy in the newborn; (2) Elaboration of the device consisted of items related to its assembly and structuring; (3) Operation of the medical device, including specific schedules related to the execution of the procedure; (4) Performing tests with simulating the purpose of volume replacement in the total cycle performed in the exsanguine transfusion procedure. The results showed that it was possible to assemble, reproduce, and implement the automation of the device developed for the exsanguine transfusion procedure in a practical. Also, the procedure presented security and effectiveness in the clinical treatment related to HDN.
\end{abstract}

Keywords: Newborn Hemolytic Disease. Fetal Erythroblastosis. Neonatal Jaundice. Kernicterus. Total Transfusion.

Abbreviations: HDN: Hemolytic disease of the newborn. HDFN: Hemolytic disease of the fetus and newborn. $\mathrm{Rh}(\mathrm{D})$ : Rhesus factor $\mathrm{D}$.

\section{Introduction}

Fetal erythroblastosis or hemolytic disease of the newborn is a typical disease caused by the incompatibility between the $\mathrm{Rh}$ Factor of maternal and fetal blood. In this pathology, the baby $\mathrm{Rh}$ factor is decisive, and the $\mathrm{Rh}$ factor of the mother is negative, which leads to the production of anti-Rh antibodies in the maternal organism to combat the $\mathrm{Rh}$ agent of the fetus. Currently, even with the introduction of anti-Rh (RhIg) immunoglobulin, Rh (D) incompatibility still happens [1].

Among Caucasians and Afro-Americans descendants in the United States, the incompatibility concerning $\mathrm{Rh}$ (D) antigen

Received on 10 July 2020; revised 22 August 2020.

Address for correspondence: Ms. Patrícia Frota. Maternidade de Referência José Maria de Magalhães Neto. Rua Marquês de Maricá, s/n - Pau Miúdo. Zip Code: 40.310-740. Salvador, Bahia, Brazil. E-mail address: quariguazypaty@gmail.com.

J Bioeng. Tech. Appl. Health

2020;3(3):279-287.

(C) 2020 by SENAI CIMATEC. All rights reserved. occurs in approximately $10 \%$ of all pregnancies; $60 \%-70 \%$ of $\mathrm{Rh}$ (D) negative women happens with an $\mathrm{Rh}(\mathrm{D})$ positive baby. Before the routine use of Rh Immune Globulin (RhIG) prophylaxis in obstetric practice, approximately $15 \%$ of D-negative women develop anti-D. Anti-D is still one of the most common antibodies associated with HDN, occurring around 1 in 1,000 births, although the rate has decreased dramatically since 1968 with the introduction of RhIG. These statistics reflect the decline in the incidence of anti-D, but they underestimate the current contribution of HDN because early intrauterine deaths are not detected, whereas neonatal deaths can be attributed to other prevalent complications [2].

Also, during pregnancy, it is recommended to identify the maternal blood type for the ABO and RhD system, and screen if irregular antierythrocyte antibodies are being produced. The diagnosis of isoimmunization by the Rh system is made based on the detection of anti-D antibodies in maternal serum. The Indirect Coombs test is the gold-standard method for diagnostic purposes, 
as it is the most accurate to determine antibody titers [3].

$\mathrm{RH}$ isoimmunization begins to be studied based on previously obtained data and laboratory tests identify the presence of antibodies in the mother's blood. Treatment for isoimmunization will depend on the degree of anemia of the fetus. Nevertheless, the pregnant woman must perform the first follow-up in her prenatal care, and also to measure the possible severity of the problem in order to schedule the delivery and avoid severe fetus' sequelae.

Phototherapy is the first line of treatment for neonatal jaundice and can prevent blood transfusion. The blood transfusion is necessary when phototherapy fails to promote an adequate reduction in bilirubin levels or when the initial serum bilirubin is so high that it implies a high risk of the newborn develops Kernicterus [4].

Innovative activities in the health area are characterized by a strong interaction with the scientific industry. On the one hand, the scientific infrastructure is the source of a flow of information that supports the emergence of innovations that affect medical practice and health such as new drugs, new equipment, new clinical procedures, new prophylactic measures, and further knowledge. Still, medical practice and the performance of the health sector are the source of an inverse flow of information and constitute a vast and growing repository of issues, empirical findings, and successful practices that need to be explained and understood [5].

So, this study aims to discuss the topic of HDN to present the characteristics of the disease, therapeutic solutions used, as well as the presentation devices that can assist the treatment of exsanguine transfusion using innovative methods associated with technologies in health [6]. The update in this field seeks to add safety to the procedures and confidence of the patients associated with a lower psychological, exhaustive, and manual burden for health professionals.

\section{Literature Review}

History of Immunohematology in the Discovery of Hemolytic Disease in the Newborn - HDN

Although changes in the fetus and newborn have been observed since the 17 th century concerning issues oh HDN, the theme highlights in 1939 when Levine and Stetson published the results from a transfusion reaction of the husband's blood to a woman after delivery. These authors postulated that the mother had been immunized against the fetus' antigen by the father. In 1940, Landsteiner and Wiener carried out experiments to immunize rabbits and guinea pigs against erythrocytes of rhesus monkeys. Using the serum from these experiments, Levine demonstrated that the mother who had suffered the transfusion reaction was rhesus-negative, and the father, rhesus-positive. Also, the mother's serum agglutinated the father's erythrocytes [7].

Few studies analyze the incidence of perinatal hemolytic disease in Brazil, a condition caused by the hemolytic action of maternal antibodies against fetal red blood cell antigens, which results in fetal and neonatal hemolytic anemia. Although the introduction of anti-Rh (D) immunoglobulin in clinical practice has considerably reduced the incidence of the disease, some evidence suggests that the number of cases is still high, even in developed countries [8].

\section{Hemolytic Disease of the Newborn - HDN}

The transplacental passage of antibodies against fetus blood cells from the 10th week of gestation can cause premature destruction of these cells (hemolysis), leading to fetal anemia. This condition is called HDN. HDN is the most complex of the clinical forms of IgG-mediated hemolysis because it involves the production of antibodies in one individual and cell destruction in another. Normally, the maternal and fetal blood systems do not mix, but transplacental maternal-fetal bleeding can occur. This bleeding 
happens most of the time during childbirth, but it can occur spontaneously during the pregnancy, especially in the $3^{\text {rd }}$ trimester or after invasive procedures or miscarriages $[3,5]$. HDN can be classified as a mild, moderate, or severe condition depending on its clinical manifestations. It is a mild disease because if it presents mild anemia with or without jaundice. In moderate cases, the child has severe anemia and jaundice and may present hepatosplenomegaly, edema, and pallor. In severe cases, there is intrauterine death from liver dysfunction and hydrops fetal. The diagnosis can be made or during pregnancy or after birth. The results are concluded by tests such as the search for antibodies fixed on the red blood cells (Direct Antiglobulin Test - TAD); elution that serves to remove the antibodies present in the erythrocyte membrane to identify them later, serum antibody testing (Irregular Antibody Research - Indirect Coombs or PAI), among others [9].

\section{Phototherapy}

Phototherapy is a therapeutic used to treat several dermatoses [10]. Used for more than 50 years, phototherapy does not have a list of still known photodegradation mechanisms, but it is quite widespread and accepted. Its use must be concomitant with laboratory evaluations, in order to highlight the cause of hyperbilirubinemia, which is a frequent clinical problem in the neonatal period. Phototherapy increases the degradation of bilirubin in the skin by photooxidation. It is contraindicated for patients with elevated conjugated bilirubin (Figure 1). Jaundice on the skin is not a reliable means of obtaining serum levels. However, there are also treatment alternatives such as blood transfusion and the use of medications capable of accelerating metabolism and its excretion [11]. The complications caused by the use of phototherapy and its side effects are skin rash, burns, thermoregulation, effects on the retina, effects on the gastrointestinal tract, effects on the speed of cerebral blood flow, behavioral changes, and effects on the baby-family relationship and the bronze baby syndrome. In the case of $\mathrm{Rh}$ and sometimes $\mathrm{ABO}$ incompatibility, phototherapy (Figures 2 and 3) should be used as an adjunct, and blood transfusion is the most appropriate treatment [12].

\section{Blood Transfusion}

Blood transfusion was the first successful therapy to treat severe neonatal jaundice. Although blood transfusion is considered a safe procedure, it is not without risks and has mortality rates ranging between $0.5 \%$ and $3.3 \%$. The bilirubin levels to indicate blood transfusion remains controversial since the incidence of bilirubin encephalopathy also depends on other variables such as gestational age, the presence or absence of hemolysis, and the newborn's clinical condition. Current recommendations for performing blood transfusion are based on the balance between risks for the occurrence of encephalopathy and adverse events related to the procedure [13]. The decision to initiate blood transfusion is due to the urgency of lowering circulating bilirubin values and promoting the turnover between them and the tissues to not affect the newborn's central nervous system [14].

Adverse events may occur after the procedure. However, it is not certain that all clinical conditions can be considered adverse events attributed to the procedure, since, in most cases, such events occurred in patients with unstable clinical conditions before blood transfusion [15].

\section{Bilirubin Encephalopathy (Kernicterus)}

The pathogenesis of kernicterus is highly complex, and its risk is related to many factors, such as the low water solubility of bilirubin and its tendency to undergo aggregation and precipitate the physiological ph (acid), the ability of bilirubin to freely cross the barrier hematoencephalic, together with prematurity, as well as metabolic acidosis, hypoglycemia, hypothermia, and sepsis can aggravate the neurotoxicity of bilirubin even at lower levels [16]. 
Figure 1. Bilirubin metabolism of the newborn and fetus.

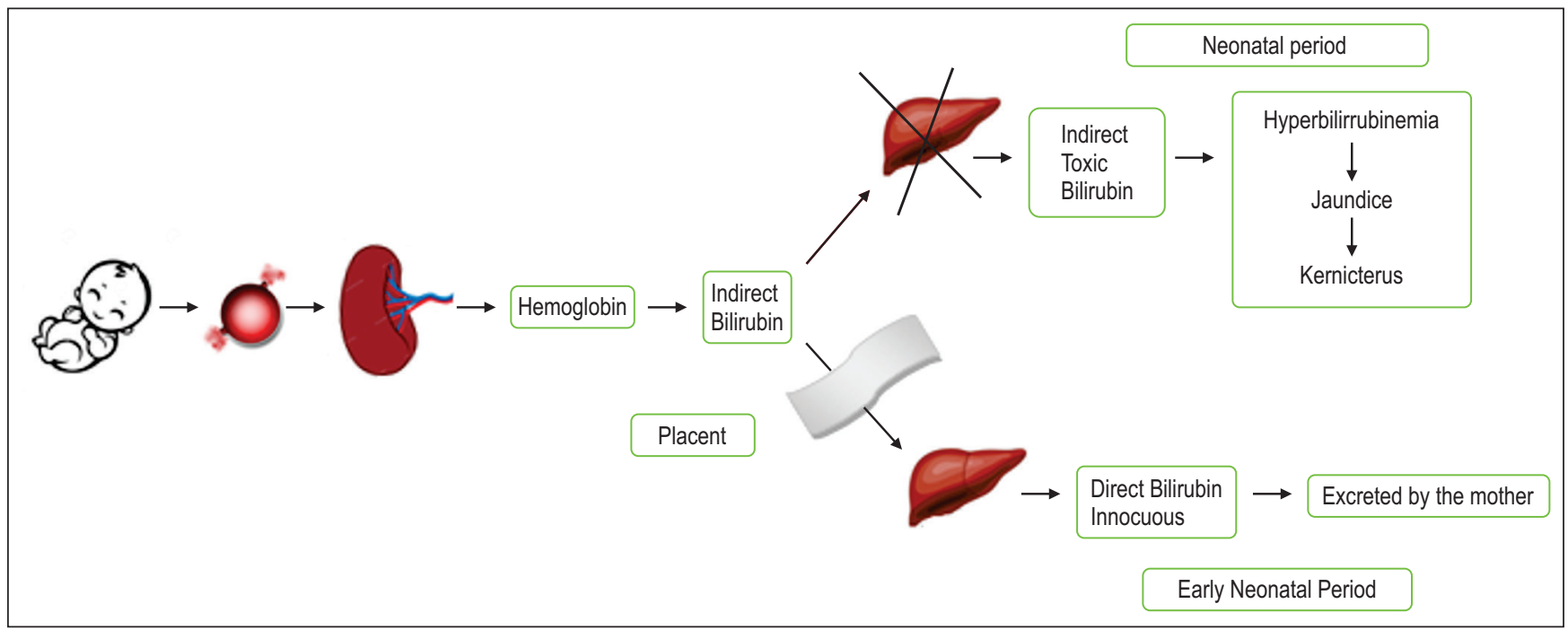

Figure 2. Newborn using portable phototherapy.

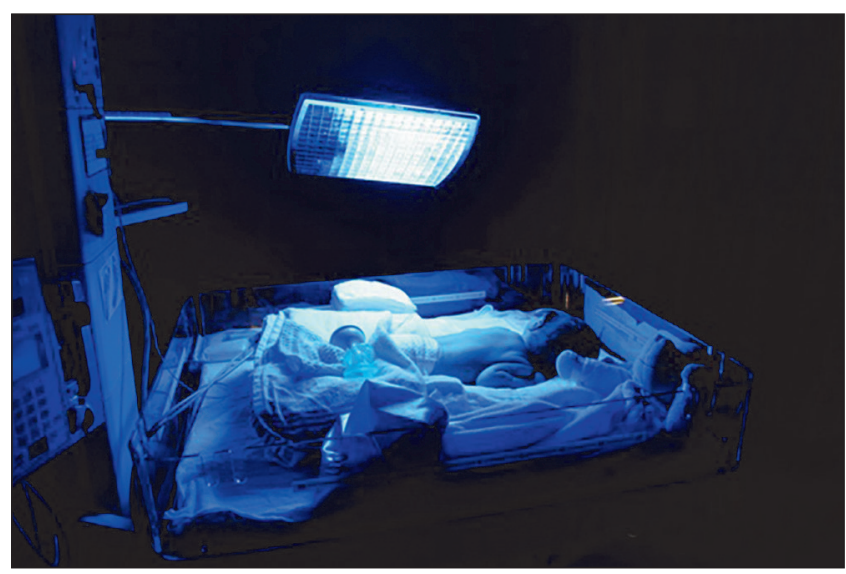

\section{Innovation and Technology in Health}

Technology can be generically defined as applied knowledge. In health areas, this applied knowledge allows the prevention, diagnosis, and treatment of diseases and the rehabilitation of their consequences [17]. The health field has been sensitive to technological incorporation for therapeutic, diagnostic, and life maintenance purposes, using the knowledge and products of computer science, new equipment, and materials. However, it has been less aggressive in the use of innovations of the non-material type, especially of the innovations in
Figure 3. Newborn in the use of phototherapy in incubators.

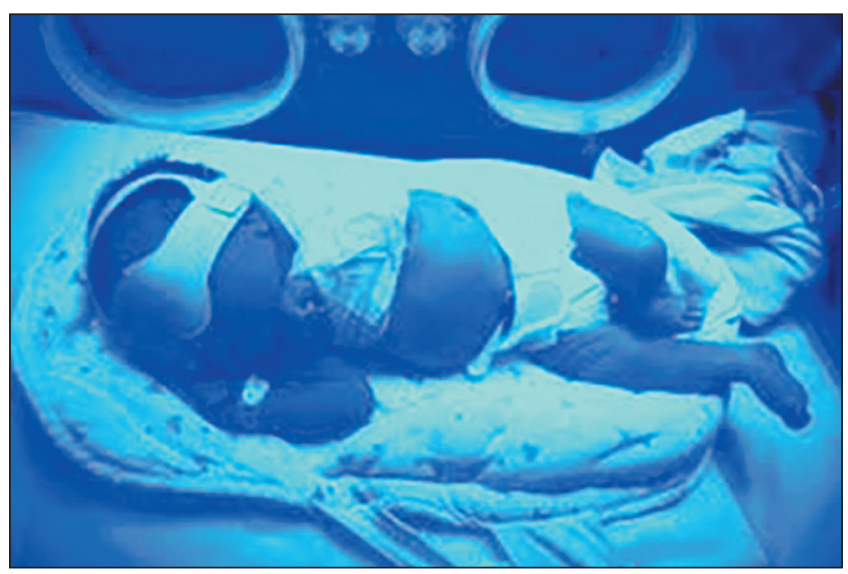

the field of the organization and work relations [18]. Technology is not only seen as a tangible product but as a result of work that involves a set of abstract or concrete actions that have a specific purpose in health care [19]. For a better understanding and development of this innovative device, it was necessary to study theoretical concepts relevant to the manuals procedures used today to perform the transfusion exsanguine technique. Although some of the concepts require a more extensive detail of the theme for its full understanding, to maintain the main focus, only the features most related to the device were presented here. 


\section{Materials and Methods}

This study was divided into four phases:

1) The selection of published scientific articles related to the topic in Portuguese and English, and exploratory analysis for the materials' contents (articles that addressed other pathologies associated with HDN were excluded from this work).

2) The development of the prototype related to the automated device for the procedure of blood transfusion.

3) The elaboration of the device and its essential materials such as disposable bags of blood components, equipment, syringes, "tree-ways", as well as a 3D printer of small supports, bearings, and specific compartments for positioning, adaptation, and fitting of these referred materials. In this phase, the device was inserted in the experimental bench to simulate the infusion of blood in the newborn. The programming of the rotation of the "tree-ways" valves related to the process of infusion and disposal left the theoretical conception of the system for practical experimentation, allowing not only the evidence of the functioning of the device but also the programming of the infusion time and the previous determination the volume of the infused blood component. Positively, the concern regarding safety in the correct disposal of the blood component generated at the end of the blood transfusion procedure was aroused.

4) Tests were performed with the system. They were processed with the inclusion of bags of blood components adapted to the system, where inside there were colored liquid for a reliable demonstration of the procedure and its functioning. Also, START / ON, PAUSE, and STOP programs were developed and included for the device's operation and safety, in case of possible intrinsic complications, which could occur during the automated procedure, ensuring the integrity of the newborn. $\underline{\text { Record }}$

The device's registration initially began with the application to the Center for Technological Innovation (NIT) of the Bahiana School of Medicine and Public Health. In response, the NIT approved the "Intellectual Protection Feasibility Opinion", now entitled "Automated blood transfusion device". According to the opinion in the thorough search for priority, a total of seven patents were found associated with the chosen theme, which could be registered as processing to perform blood transfusion, but not for the same purpose. Of the evaluated works, only one was identified as a control system in the activity related to the automation of the blood transfusion procedure [patent number 6 (PI 0404065-1 A). Filing Date: 9/21/2004. dissertation].

\section{Conception}

The experimental device described in this study is developed as an alternative infuser with four cycles that can replace the manual work currently carried out by the medical team in the blood transfusion procedure. The main purpose of this device is to ensure that the volume and speed of infusion and blood withdrawal of the patient are uniform and that the sequencing of the valves is performed with optimal reliability. These guarantees protect the patient concerning noncollapse (narrowing of the blood vessel walls when subjected to a negative pressure) of the umbilical vein of access, hemolysis in the blood infusion, and reduction of the time to perform the procedure. The prototype was initially tested with bags containing pigmented water with different colors, facilitating the visualization of the "blood" exchange.

\section{Detailed Device Composition}

The experimental device consists of a mechanical structure (fixed and mobile part) in a polymeric material, a spindle, and two steel rails. One end of the spindle is coupled to a bearing attached to the fixed part and the other coupled 
to the stepper motor that converts the rotational movement of the motor into a linear movement causing the mobile part to displace the syringe plunger (Figure 4).

Another part of the device is composed of two servo motors fixed in a rigid structure and coupled to two supports for connecting the "three ways" valves (Figure 5).

The device's electronic circuit consists of an Arduino Uno, an A4988 stepper motor driver, power supply, communication cable, and other electronic components (LEDs, resistors, buttons, among others). All components were installed on a test plate for assembling electronic circuits (protoboard) (Figure 6).

The power supply to the circuit (12VDC) and the communication cable is used as an interface between the computer and the Arduino to download the program. This cable can also be used as a power source for the Arduino. The servo motor and stepper motor cables are responsible for the communication between them and the electronic circuit board (Protoboard). The program that controls the Arduino terminals and actions can be developed using the software called Arduino IDE (available at https://.arduino.cc). The software is free and its programming language is wiring, derived from the $\mathrm{C} / \mathrm{C}++$ language.

\section{Device Commands}

The commands inserted for the operation of the device were ON / START, STOP, and PAUSE. The ON / START button when pressed initiates the blood transfusion procedure. At any time during the experiment, if the STOP button is pressed, the blood in the syringe - from the RN or donor - is discarded and the device immediately stops. Also, at any time during the experiment, if the PAUSE button is pressed, the device stops and, if pressed again, the device returns to work from the point where it stopped. The PAUSE button can be used at any time if the user wants to pause the procedure to make any adjustments or any other action. STOP should be pressed in an emergency. A dropper sensor indicates when the donor bag is empty and the device automatically turns off, ending the blood transfusion procedure.

\section{Experimental Procedure}

The experimental procedure had the purpose of verifying the possibility to replace the technique of blood transfusion procedure by the exchange of the newborn's body blood volume for the volume of the blood component bag. The experiment took

Figure 4. The mechanical structure of the device.

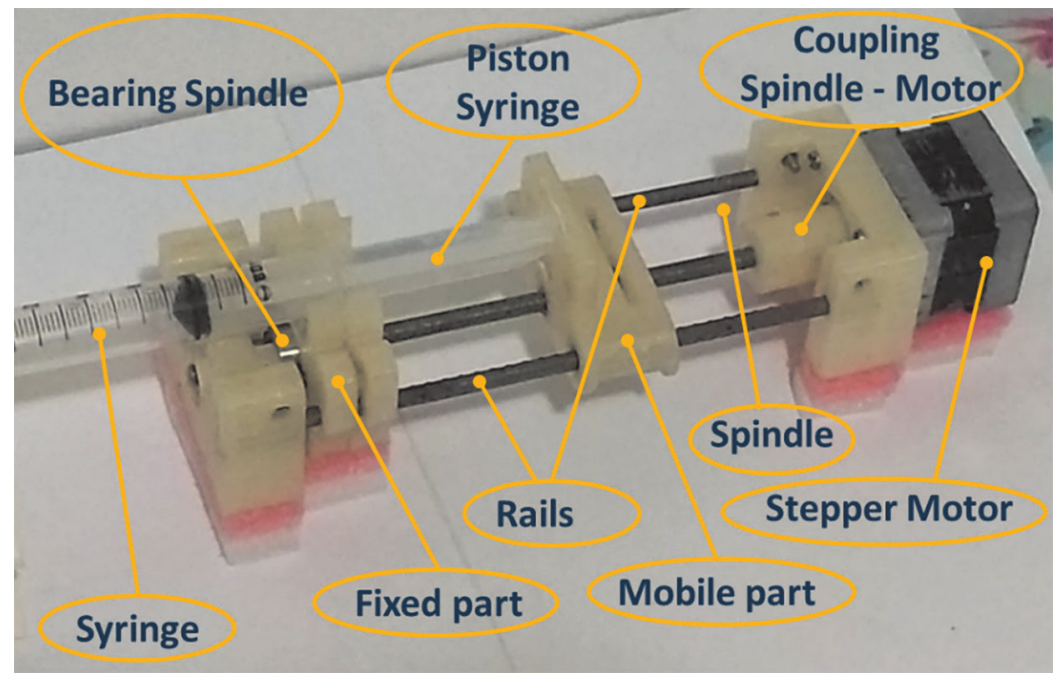


Figure 5. Rotating structure of the device.

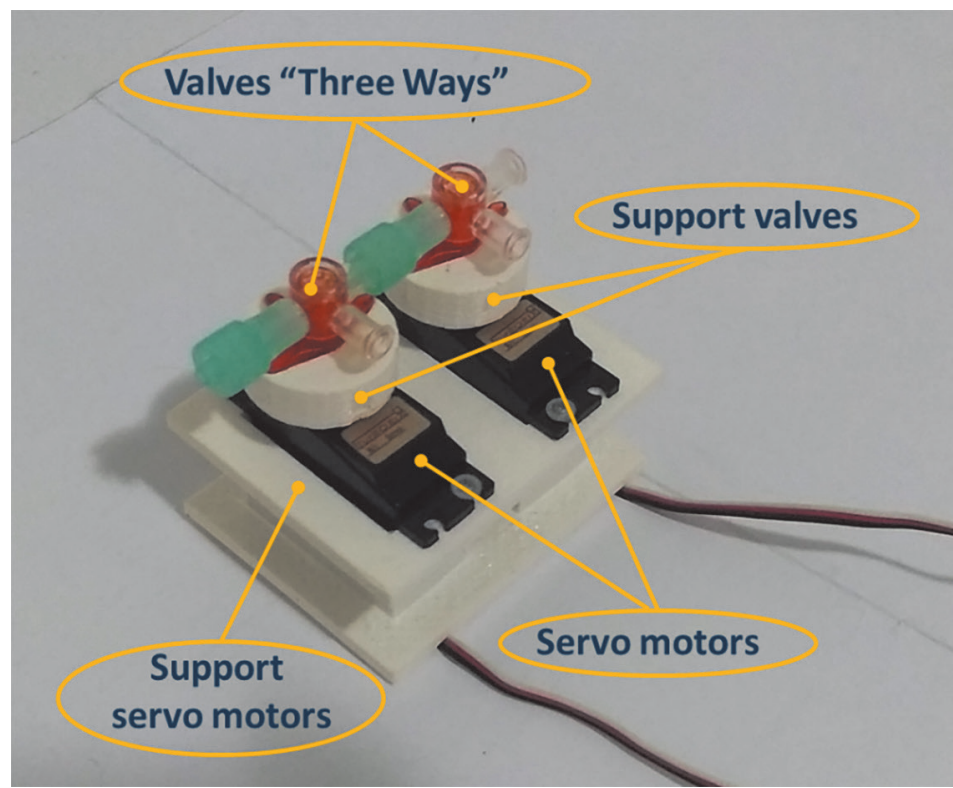

Figure 6. The electronic circuit of the device.

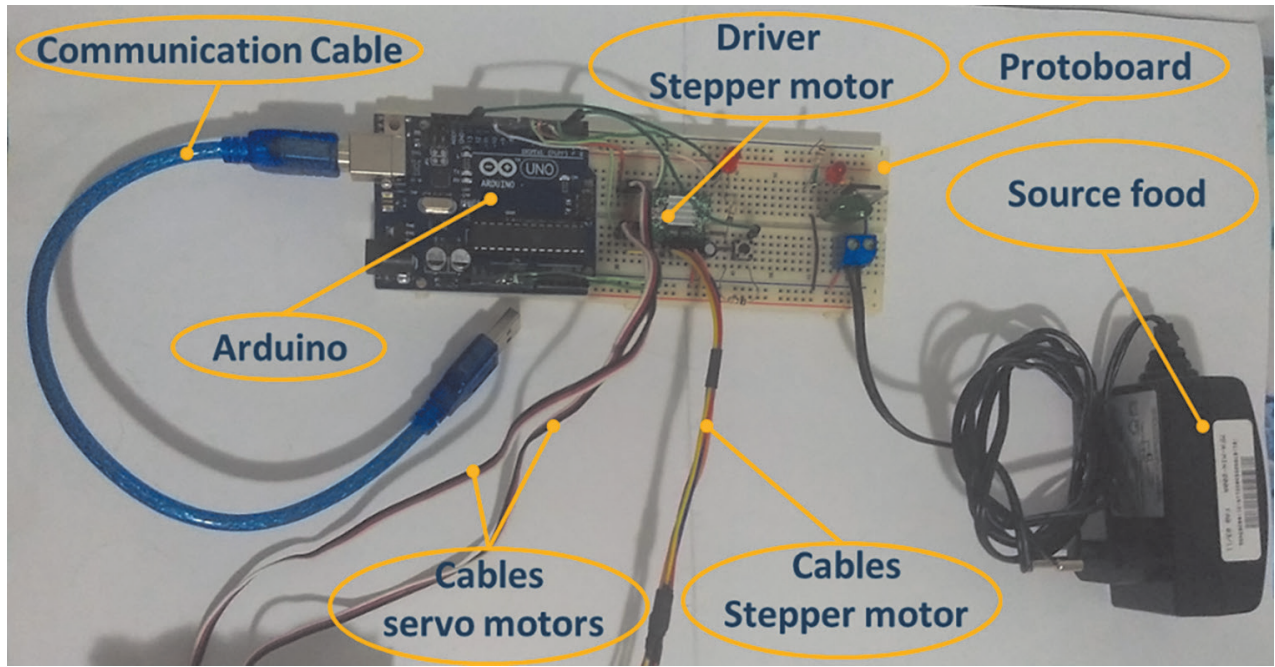

place on a test bench and the procedure time was divided into four cycles: a) time spent in aspirating the donor bag; b) time spent infusing blood in the newborn; c) time spent in aspirating the newborn's blood; d) time spent on blood disposal. It is worth mentioning that cycles "a" and " $\mathrm{d}$ " were programmed to be faster than cycles " $b$ " and "c". The cycle "b" and "c" were directly related to the withdrawal and infusion in the newborn. The tests were carried out using yellow and red-pigmented water, in which the yellow color represented the blood of the newborn and the red color represented the blood of the donor.

\section{$\underline{\text { Test Results }}$}

After carrying out the tests, the discard bags and the NB went orange, due to the mixture of yellow 
and red pigments. Thus, it was demonstrated that there was a partial exchange of the NB's blood as expected, confirming the effectiveness of the automated device. The total time to perform the procedure, from the ON / START command until the end of the donor bag's content, was $35 \mathrm{~min}$ for a volume of $300 \mathrm{~mL}$ contained in the red bag representing the donor. The time was significantly shorter compared to the time of the procedure performed manually, which was achieved by adjusting the time in the stages of aspiration of the donor bag and blood disposal, preserving for safety the time in the phases of withdrawal and infusion as recommended by the Ministry of Health.

\section{Results}

This study resulted in automated device development, as an alternative flows in four cycles, with the potential to replace the manual procedure of blood transfusion therapy.

\section{Discussion}

This study presented a new device related to the procedure of blood transfusion in the therapeutic conduct of HDN. Currently, the manual procedure represents exhaustive, tense, and unsafe conduct by health professionals since it involves many sorts of situations such as calculating the volume of the infused blood component, the excessive volume of incorrect exchanges, the speed of infusion and blood withdrawal for disposal, control on the correct rotation of the valves, the records related to each movement performed adapted to the syringe, the possibility of the collapse of the umbilical vein at the time of blood aspiration, the possibility of pulmonary embolism related to the presence of air in the syringe during the procedure, care with handling the catheter in the umbilical stump and the possibility of infection caused by the invasive procedure. Many studies point out that the blood transfusion procedure is not without risks. Bradycardia, apnea, hypoglycemia, hypocalcemia, hyponatremia, and hypernatremia are mentioned as complications related to this volume replacement technique [20]. The most serious of the morbidities include symptomatic hypocalcemia, apnea, and bradycardia with cyanosis requiring resuscitation. These complications are common enough that the exchange transfusion, even in healthy newborns, should be performed only in Neonatal Intensive Care Units prepared to respond to these events [21].

At a time when many neonatal care providers have more experience with advanced therapies, such as high frequency of ventilation, dialysis, extracorporeal membrane oxygenation than with blood transfusion, a standardized protocol for performing the procedure can be an important tool to decrease the number of related adverse events [22].

Although an automated procedure does not eliminate the complications of blood transfusion and is likely to bring new inconveniences, it is believed that this device will bring greater safety to the team, freeing them from a tiring and repetitive routine, reducing the tension deposited during the manual procedure. So, the device can assume these actions providing more time in the humanized care to the newborn during and after the procedure such as the monitoring of the newborn's biochemical rates, heating of the crib, and use of post-procedure phototherapy.

Like other automated equipment, such as infusion by peristaltic pumps, safety mechanisms incorporated in the equipment guarantee total control of the volume to be infused per cycle, programmed time of infusion and withdrawal, flow control and speed of independent discharges, as well as a precaution against air inlet, infusion pressure sensor, and obstruction detector. Another proposal presented of the device is related to the reduction in the manipulation of venous access, decreasing the risk of possible infections. Also, the concern about the proper disposal of the blood drawn is a positive point of the device regarding the waste management plan in health services.

So, this functional device is an alternative infuser of four cycles and intends to carry out the automated procedure of blood transfusion. 
Primarily there was the creation of a prototype, and in later studies, it will be tested in newborns proving its safety and effectiveness in specific hemotherapeutic treatments.

\section{Conclusion}

We concluded that it is possible to replace the manual and rudimentary procedures of the blood transfusion process with the development of an automated device, an alternative four-cycle infuser using three-way valves, Arduino logic, blood component bag, a disposal bag, and specific teams as integral parts of the process.

\section{References}

1. Fetal, E., Silva Da Paixão, L., \& Oliveira, M. L. (n.d.). The Efficacy of Using Human Anti-D Globulin Serum to Prevent Fetal Erythroblastosis The Effective ness of the use of the Anti-human Globulin Serum Anti-D on prevention of.

2. American Association of Blood Banks. Manual for Doctors. Pediatric Transfusion. 1a.ed. 2006.

3. Pereira, Pâmela do Carmo Mesquita. Maternal Rh isoimmunization. Prophylaxis, diagnosis and treatment: current aspects. Federal university of Bahia. Bahia Medical School, 2012.

4. Fetal anemia due to Isoimmunization RH. (n.d.). Retrieved from.

5. Machado, I. N., Barini, R., Perinatal Hemolytic Disease: current aspects Perinatal hemolytic disease: currentas pects. See. Ciênc. Méd., Campinas, Jan./Feb., 2006.

6. Albuquerque, E. D. M. E., Souza, S. G. A. D., \& Baessa, A. R. (2004). Health research and innovation: a discussion based on the literature on technology economics. Ciência \& Saúde Coletiva, 9, 277-294.

7. Harmening, M. Denise. Modern Techniques in Blood Bank and Transfusion. 4th. ed.2006.

8. Lobato, G., Reichenheim, M. E., \& Coeli, C. M. (2008). Hospital information system of the Unified Health System (SIH-SUS): a preliminary assessment of its performance in monitoring perinatal hemolytic disease Rh (D). Public Health Notebooks, 24 (3), 606-614.

9. De, P., Taveira, A., \& Rios, A., (n.d.). Hemolytic Disease of the Newborn caused by antigen and Hemolytic disease of the newborn caused by antigen.
10. Duarte, I., Buense, R., \& Kobata, C. (2006). Phototherapy. Anais Brasileiros de Dermatologia, 81 (1), 74-82.

11. Moreira VL, Sacramento CB, Alecrin AF et al. Neonatal Jaundice and phototherapy: the contribution of nurses to the effectiveness of treatment. ISSN 2175-5361.

12. Gomes, M. P., Silva, D., De, M., \& Pereira Do Nascimento, J. (n.d.). Phototherapy in the treatment of neonatal hyperbilirubinemia.

13. Amaral Sá, C. M., Cristina Santos, M. P., de Carvalho, M., \& Elisabeth Moreira, M. L. (n.d.). Adverse events associated with blood transfusion in perinatal hemolytic disease: ten-year experience Adverse events related to exchange transfusion in new born in fants with hemolytic disease: has years of experience.

14. Malono, J. Nabais, I. Cohen, A. Fraga, G. Gonçalves, S. Hemolytic Disease of the Newborn; Review article. Consensus in Neonatology.

15. Sá CAM, Santos MCP, Carvalho M, M. M. (2009). Adverse events associated with exsanguineotransfusion in perinatal hemolytic disease: ten years' experience Adverse events related to exchange transfusion in newborn infants with hemolytic disease: ten years of experience. Rev Paul Pediatr, 27 (2), 168-72.

16. Vinhal, R. M., Cardoso, T. R. C., \& Formiga, C. K. M. R. (2009). Neonatal jaundice and kernicterus: knowing to prevent neonatal. Movimenta Magazine, 2 (3), 93101.

17. Leila, C., Garcia, P., Garcia, L. P., \& Zanetti-Ramos, B. G. (2004). Health service waste management: a question of biosafety.

18. Santos, Z. M. D. S. A., fleet, m. A., \& martins, a. B. T. (2016). Health technologies: from the theoretical approach to construction and application in the care setting.

19. Trindade, D. L., Lorenzetti, J., Trindade, L. L., Pires, D. E. P., \& Souza, F. R. R. (2012). Technology, technological innovation and health: a necessary reflection. Text Contexto Enferm, 21 (2), 432-9.

20. Women's Beneficent Society Hospital SírioLibanês. Guide to hemotherapy treatments, Guide to Hemotherapy treatments (2010).

21. Behjati, S., Sagheb, S., Aryasepehr, S., \& Yaghmai, B. (2009). Adverse events associated with neonatal exchange transfusion for hyperbilirubinemia. Indian $\mathrm{J}$ Pediatr, 76 (1), 83-85.

22. Orme, R. L. E., \& Eades, S. M. (1968). Perforation of the Bowel in the Newborn as a Complication of Exchange Transfusion. British Medical Journal, 4 (5627), 349-351. 